\title{
RESTAURAÇÃO DE ÁREA ALTERADA: O REFLORESTAMENTO COM ESPÉCIES NATIVAS E A REGENERAÇÃO NATURAL
}

\author{
José Eduardo de Arruda Bertoni ${ }^{1}$ \\ Ernesto Pedro Dickfeldt ${ }^{2}$ \\ Walter José Siqueira
}

\section{RESUMO}

Uma área alterada de floresta foi restaurada através da retirada de cipós, plantio de mudas de 12 espécies nativas e manejo adequado. As mudas plantadas foram medidas de dois em dois anos, até o sexto ano. Para avaliar o desenvolvimento das mudas plantadas e a regeneração natural, no quarto ano de implantação foi realizado um levantamento pelo método de parcelas. Foram encontradas 41 espécies, sendo 12 plantadas e 29 da regeneração natural. Em 22 parcelas $\left(550 \mathrm{~m}^{2}\right)$ foram amostrados 344 indivíduos, sendo $117(34,0 \%)$ das espécies plantadas, 207 (60,2\%) das espécies da regeneração natural e $20(5,8 \%)$ plantas mortas. A restauração foi favorecida pelo manejo utilizado, enriquecimento com espécies nativas, presença do banco de sementes e pelas fontes de dispersão. No quarto ano após a implantação a área já apresentava fisionomia de capoeira em franca recuperação.

Palavras-chave: restauração, regeneração natural, silvicultura, espécies nativas

\section{Introdução}

\footnotetext{
${ }^{1}$ Pesquisador Científico-Instituto Agronômico de Campinas-SAA-SP

${ }^{2}$ Biólogo-InstitutoFlorestal-SMA-SP
} 


\section{FÓRUM AMBIENTAL DA ALTA PAULISTA}

Volume VI - Ano 2010

Para avaliar os resultados do reflorestamento e da recomposição natural da área manejada, realizou-se:

a) medições dos diâmetros e alturas de dois em dois anos;

b) levantamento da composição e estrutura da área em recuperação depois de quatro anos

Tabela 1- Espécies arbóreas nativas plantadas na área a ser restaurada no Parque Estadual de Porto Ferreira (SP), em ordem alfabética por família, nome comum e grupo ecológico a que pertencem $(\mathrm{P}=$ pioneira, $\mathrm{S}=$ secundária e $\mathrm{C}=$ clímax $)$.

\begin{tabular}{|c|c|c|}
\hline FAMILIAS E ESPECIES & NOME COMUM & GRUPO ECOLOGICO \\
\hline ANACARDIACEAE & & $\mathrm{S}$ \\
\hline 1- Miracrodruon urundeuva Fr.All. & Aroeira & \\
\hline BORAGINACEAE & & S \\
\hline 2- Patagonula americana L. & Guaiuvira & \\
\hline CECROPIACEAE & & $P$ \\
\hline 3- Cecropia glaziovii Miq. & Embauva-da-mata & $P$ \\
\hline $\begin{array}{l}\text { 4- Cecropia pachystachia Trec. } \\
\text { LAURACEAE }\end{array}$ & Embauva-do-brejo & $\mathrm{c}$ \\
\hline 5- Ocotea puberula (Rich.)Nees & Canela & \\
\hline MELIACEAE & & S \\
\hline 6- Cedrela odorata L. & Cedro-do-brejo & \\
\hline MIMOSOIDEAE & & $\mathrm{S}$ \\
\hline 7- Albizzia hasslerii (Chodat)Burr. & Farinha-sêca & \\
\hline MORACEAE & & S \\
\hline $\begin{array}{l}\text { 8- Fícus sp } \\
\text { 9- Jacaratia spinosa (Aubl.)DC }\end{array}$ & $\begin{array}{c}\text { Figueira } \\
\text { Jaracatiá }\end{array}$ & $S$ \\
\hline PALMAE & & S \\
\hline $\begin{array}{l}\text { 10- Syagrus oleracea (Mart.) Becc. } \\
\text { ULMACEAE }\end{array}$ & Guairoba & $P$ \\
\hline 11- Trema micrantha (L.) Blum. & Polveiro & \\
\hline VERBENACEAE & & S \\
\hline 12- Vitex cymosa Bert. & Tarumã & \\
\hline
\end{tabular}

Amostras de solo foram retiradas em 5 pontos dentro da área estudada, nas profundidades de 0 - 20cm e 40 - 60cm, perfazendo 2 amostras compostas (Tabela 2). As mudas plantadas não receberam nenhum tipo de adubação ou corretivo de acidez do solo, pois era intenção avaliar o desenvolvimento das mesmas nas condições locais e sem gastos com fertilizantes. 
TABELA 2. Resultados da análise de solo da área estudada no Parque Estadual de Porto Ferreira (SP).

\begin{tabular}{ccccccccccc}
\hline Profundidade & M.O & pH & P & K & Ca & Mg & H+Al & S.B & CTC & V\% \\
\hline $\mathbf{0 - 2 0}$ & 28 & 4,6 & 8 & 2,5 & 18 & 5 & 42 & 25,5 & 67,8 & 38 \\
\hline $\mathbf{4 0 - 6 0}$ & 11 & 4,5 & 3 & 1,6 & 6 & 2 & 38 & 9,6 & 47,7 & 20
\end{tabular}

As atividades de manejo efetuadas periòdicamente foram: coroação somente das mudas plantadas, corte de cipós duas vezes por ano, retirada de gramíneas e combate às formigas saúvas. As mudas de espécies nativas da regeneração, nascidas posteriormente, foram preservadas. No $2^{\circ}$ ano foi feito o replantio de falhas, a coroação até $\circ 3^{\circ}$ ano, o controle de formigas saúvas até o $4^{\circ}$ ano e o corte de cipós até o $6^{\circ}$ ano (Tabela 3). O acompanhamento do desenvolvimento das mudas foi realizado através de medidas de alturas e diâmetros na base aos 2, 4 e 6, anos de idade. A regeneração natural teve início logo em seguida à retirada dos cipós e plantio das mudas, observações e anotações feitas periòdicamente.

TABELA 3. Atividades de implantação e manejo da área alterada no Parque Estadual de Porto Ferreira (SP) após a roçada.

\begin{tabular}{|c|c|c|c|c|c|c|}
\hline \multirow[t]{2}{*}{ Atividades } & \multicolumn{6}{|c|}{ Período (Anos) } \\
\hline & $1^{\underline{ }}$ & $2^{\underline{4}}$ & $3^{\underline{m}}$ & $4^{-}$ & $5^{\underline{5}}$ & $6^{\underline{2}}$ \\
\hline Plantio & $X$ & & & & & \\
\hline Replantio & & $x$ & & & & \\
\hline Contagem & & $x$ & & $x$ & & \\
\hline Medição & & $x$ & & $x$ & & $x$ \\
\hline Coroação das mudas & $x$ & $x$ & $x$ & & & \\
\hline Corte de cipós & $x$ & $x$ & $x$ & $x$ & $x$ & $x$ \\
\hline Combate a saúvas & $x$ & $x$ & $x$ & $x$ & & \\
\hline
\end{tabular}


No estudo da regeneração natural foram demarcadas 22 parcelas de $5 \times 5 \mathrm{~m}\left(25 \mathrm{~m}^{2}\right)$ dentro da clareira, totalizando $550 \mathrm{~m}^{2}$, representando cerca de $70 \%$ da área total descontando-se o entorno, incluindo todos os indivíduos arbóreos e arbustivos, plantados ou da regeneração natural, acima de $1 \mathrm{~m}$ de altura independente do diâmetro medido na base do caule. Para analisar e comparar as alturas e diâmetros das espécies plantadas aplicou-se o teste de médias SANEST ( Zonta \& Machado, 1998 ), com delineamento experimental inteiramente casualizado e significância ao nível de $5 \%$. No levantamento florístico, os parâmetros fitossociológicos foram obtidos através do programa FITOPAC (Shepherd, 1996).

\section{Resultados e discussão}

\section{1- O plantio de espécies nativas}

Após o plantio e replante das falhas no primeiro ano, iniciou-se no segundo ano as medições e contagens das mudas mortas seguindo o "croqui". As medidas de alturas e diâmetros foram realizadas de dois em dois anos até o sexto ano. A tabela 4 apresenta a porcentagem de sobrevivência das espécies plantadas.

Tabela 4. Espécies florestais nativas plantadas na área a ser restaurada no Parque Estadual de Porto Ferreira (SP), e as respectivas porcentagens de sobrevivência no $2^{\circ}$, $4^{\circ}$ e $6^{\circ}$ ano após a implantação.

\begin{tabular}{llcc}
\hline \multicolumn{1}{c}{ ESPECIES } & \multicolumn{2}{l}{} \\
\hline \multicolumn{1}{c}{$\mathbf{2}^{\circ}$ ano } & $\mathbf{4}^{\circ}$ ano & $\mathbf{6}^{\circ}$ ano \\
\hline Cecropia glaziovii & 100,00 & 100,00 & 100,00 \\
Albizia hasslerii & 100,00 & 100,00 & 100,00 \\
Miracrodruon urundeuva & 100,00 & 95,93 & 91,87 \\
Syagrus aleracea & 100,00 & 90,00 & 90,00 \\
Patagonula americana & 100,00 & 100,00 & 86,67 \\
Cecropia pachystachya & 73,33 & 73,33 & 78,33
\end{tabular}


Ficus sp

Cedrela odorata

Vitex cymosa

Jacaratia spinosa

Trema micrantha

Ocotea puberula
90,00

70,00

60,00

51,40

85,00

20,00
72,73

72,73

70,00

40,00

16,22

3,33

2,63

Das doze espécies plantadas, oito tiveram sobrevivência igual ou acima de $70 \%$ no sexto ano de avaliação. A espécie Vitex cymosa teve sobrevivência razoável de 40\%, mas as três ultimas não tiveram muito sucesso. As quatro espécies que mais se destacaram em sobrevivência foram: Cecropia glaziovii (100\%), Albizzia hasslerii (100\%), Miracrodruon urudeuva $(91,1 \%)$ e Syagrus oleracea $(90 \%)$. As diferenças de sobrevivência entre as espécies plantadas, certamente foram devido às características do desenvolvimento da espécie, solo, fatores que interagiram na dinâmica da regeneração, tal como concorrência entre as espécies por luz, nutrientes, predação por formigas cortadeiras e outros.

A análise estatística para médias de alturas e diâmetros, aos quatro e seis anos, é apresentada na Tabela 5. O coeficiente de variação (cv ) embora tenha sido elevado $(45,73$ a $51,35 \%)$, conseguiu detectar diferença significativa $(5 \%)$ entre as diferentes espécies arbóreas plantadas. A provável razão para estes coeficientes elevados seria 0 fato das plantas terem desenvolvimentos diferentes conforme a espécie, do plantio ser heterogêneo e ainda a concorrência com as espécies pioneiras. 
Tabela 5. Análise Estatística pelo Programa SANEST (teste de médias de Duncan) para médias de alturas e diâmetros aos 4 e 6 anos, por ordem decrescente de alturas aos 6 anos, para as espécies plantadas em Porto Ferreira (SP). Médias seguidas por letras distintas diferem entre si ao nível de $5 \%$ de probabilidade. $\mathrm{H}=$ altura $(\mathrm{m}) \mathrm{D}=$ diâmetro na base $(\mathrm{cm})$ e $\mathrm{CV}=$ coeficiente de variação.

\begin{tabular}{|c|c|c|c|c|c|}
\hline & 4 anos & & & & \\
\hline & H & D & $\mathbf{H}$ & D & \\
\hline 1) Trema micrantha & $7,55 \mathrm{a}$ & & $11,50 \mathrm{a}$ & $8,50 \mathrm{a}$ & $15,25 \mathrm{a}$ \\
\hline 2) Cecropia glaziovii & $5,09 b$ & & $6,80 \mathrm{~b}$ & $7,88 a$ & $9,40 \mathrm{~b}$ \\
\hline 3) Jaracatia spinosa & $3,49 c$ & & $7,07 \mathrm{~b}$ & $6,43 a b$ & $13,33 \mathrm{a}$ \\
\hline 4) Cecropia pachystachya & $3,73 \mathrm{c}$ & & $3,45 c$ & $6,05 \mathrm{ab}$ & $5,64 \mathrm{c}$ \\
\hline 5) Albizia hasslerii & $3,72 \mathrm{c}$ & & $3,33 \mathrm{c}$ & $5,18 b$ & $4,75 \mathrm{c}$ \\
\hline 6) Syagrus oleracea & $2,62 \mathrm{~cd}$ & & $6,22 \mathrm{~b}$ & $4,20 \mathrm{bc}$ & $9,61 \mathrm{~b}$ \\
\hline 7) Miracrodruon urundeuva & $2,58 \mathrm{~cd}$ & & $2,73 \mathrm{c}$ & $3,41 \mathrm{bc}$ & $3,76 \mathrm{c}$ \\
\hline 8) Fícus sp & $1,60 \mathrm{~d}$ & & $2,00 \mathrm{c}$ & $2,97 \mathrm{bc}$ & $4,71 \mathrm{c}$ \\
\hline 9) Ocotea puberula & $1,99 \mathrm{~cd}$ & & $3,06 \mathrm{c}$ & $2,91 \mathrm{bc}$ & $4,38 \mathrm{c}$ \\
\hline 10) Cedrela odorata & $1,80 \mathrm{~cd}$ & & $2,00 \mathrm{c}$ & $2,10 \mathrm{bc}$ & $2,00 \mathrm{~cd}$ \\
\hline 11) Patagonula americana & $1,38 d$ & & $1,33 \mathrm{c}$ & $2,05 \mathrm{c}$ & $1,73 d$ \\
\hline 12) Vitex cymosa & $0,97 d$ & & $1,17 \mathrm{c}$ & $1,85 c$ & $2,00 \mathrm{~cd}$ \\
\hline
\end{tabular}

CV para $\mathrm{H}$ aos 4 anos $=45,73 \%$

CV para $\mathrm{H}$ aos 6 anos $=47,38 \%$

CV para $D$ aos 4 anos $=51,35 \%$

CV para D aos 6 anos $=49,20 \%$ 


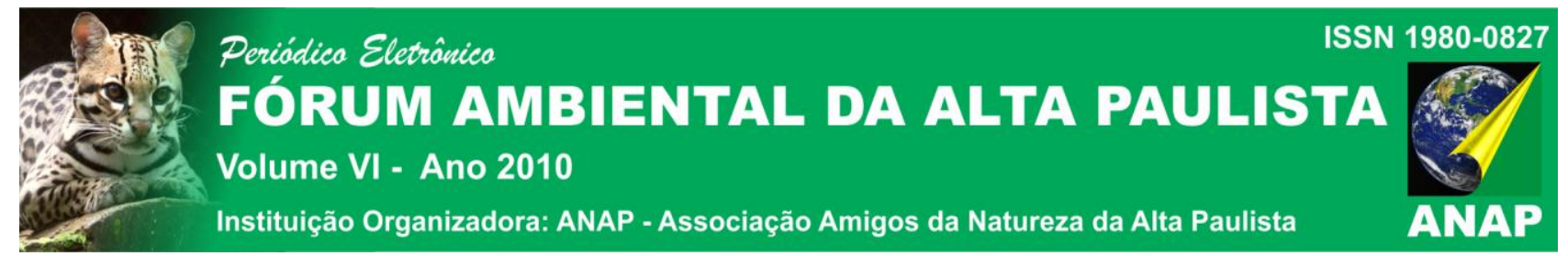


A análise estatística mostra que existem cinco grupos de espécies. As duas primeiras espécies, Trema micrantha e Cecropia glaziovii, não diferem estatisticamente entre si, bem como o segundo grupo constituído pela segunda e terceira espécie. Mas o primeiro e o segundo grupo diferem entre si. No terceiro grupo está a espécie Albizzia hasslerii sozinha, no quarto grupo estão juntas as cinco espécies seguintes sem diferenças estatísticas entre si, e no quinto grupo as duas últimas.

\section{2 - O levantamento da regeneração natural}

A Tabela 6 relaciona em ordem alfabética por família, todas as espécies encontradas na área em recuperação, tanto as plantadas como as da regeneração natural, juntamente com os nomes comuns e os grupos ecológicos a que pertencem. Apresenta as espécies amostradas, o número de indivíduos de cada espécie, alguns parâmetros fitossociológicos em ordem decrescente de IVI e as medidas, mínimas, médias e máximas, de alturas e diâmetros.

O levantamento da composição e estrutura da área manejada foi realizado para avaliar a regeneração natural e as espécies plantadas depois de quatro anos da implantação. As espécies pioneiras da regeneração natural com os maiores números de indivíduos foram: Solanum swartzianum (64), Croton floribundus (35); Cestrum calycinum (28), Aegiphila sellowiana (17); Vernonia polianthes (11) e Solanum paniculatum (7). Juntas somam 155 indivíduos, os quais representam 47,09\% do total amostrado.

No levantamento foram amostradas 41 espécies distribuídas em 24 famílias botânicas, sendo 12 espécies plantadas e mais 29 espécies da regeneração natural. Incluiu-se no levantamento as árvores mortas pela importância que representam na dinâmica florestal

Nas 22 parcelas do levantamento $\left(550 \mathrm{~m}^{2}\right)$ foram amostradas 344 indivíduos, sendo $117(34,0 \%)$ das espécies plantadas, 207 (60,2\%) das espécies da regeneração natural e 20 (5,8\%) árvores mortas. A densidade total foi 6254,55 indivíduos/ha e o Índice de Shannon $\left(\mathrm{H}^{2}\right)=2,778$.

.O método utilizado, através do plantio de mudas de espécies nativas e um manejo adequado, mostrou ser eficiente para enriquecer e acelerar a restauração. Os resultados obtidos evidenciam a possibilidade de restauração de áreas alteradas como clareiras e 


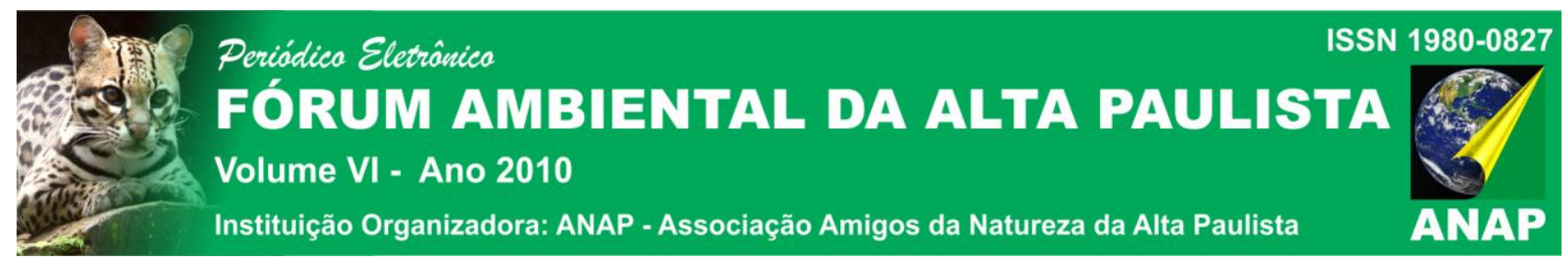

bordas de florestas, utilizando um manejo adequado e a escolha das espécies adequadas é também uma questão importante. 
Tabela 6 - Empécies arbóreas e arbustivas amostradas em área de recuperação, no 4ํano de implantação na floresta do Parque estadual de Porto Ferreira (SP) e seus parâmetros fitossociológicos, ordenado pelo $\mathrm{IVI}$, onde: $\mathrm{NI}=$ número de indivíduos; $\mathrm{DR}=$ Densidade Relativa; DoR= Dominância relativa; FR= Freqüência Relativa; IVI = Índice do Valor de Importância; IVC= Índice do Valor de Cobertura; mi=mínima; ma= máxima; me=média e * = espécies plantadas.

\begin{tabular}{|c|c|c|c|c|c|c|c|c|c|c|c|c|}
\hline \multirow[b]{2}{*}{ ESPECIES } & \multirow[b]{2}{*}{$\begin{array}{c}\text { NI } \\
(\%)\end{array}$} & \multirow[b]{2}{*}{$\begin{array}{l}\text { DR } \\
\text { (\%) }\end{array}$} & \multirow[b]{2}{*}{$\begin{array}{c}\text { DOR } \\
(\%)\end{array}$} & \multirow[b]{2}{*}{ FR } & \multirow[b]{2}{*}{ IVI } & \multirow[b]{2}{*}{ IVC } & \multicolumn{2}{|c|}{ Altura (m) } & \multicolumn{2}{|c|}{ Diâmetro (cm) } & \multirow[b]{2}{*}{ ma } & \multirow[b]{2}{*}{ me } \\
\hline & & & & & & & mi & ma & me & mi & & \\
\hline Solanum swartzianum & 64 & 18.60 & 19.96 & 11.93 & 50.50 & 38.57 & 1.1 & 7.0 & 3.9 & 0.5 & 9.0 & 4.7 \\
\hline Miracrodruon urundeuva * & 69 & 20.06 & 11.57 & 12.50 & 44.13 & 31.63 & 1.0 & 6.5 & 3.0 & 1.0 & 6.0 & 3.4 \\
\hline Croton floribundus & 35 & 10.17 & 12.17 & 6.82 & 29.17 & 22.35 & 1.0 & 8.2 & 3.9 & 0.5 & 11.0 & 4.3 \\
\hline Aegiphila sellowiana & 17 & 4.94 & 13.40 & 5.68 & 24.02 & 18.34 & 1.0 & 7.0 & 4.9 & 0.5 & 12.0 & 7.1 \\
\hline Árvores mortas & 20 & 5.81 & 6.68 & 5.68 & 18.18 & 12.50 & 1.0 & 8.0 & 4.0 & 2.0 & 9.5 & 4.7 \\
\hline Cestrum calycinum & 28 & 8.14 & 0.94 & 7.95 & 17.03 & 9.08 & 1.0 & 3.0 & 1.6 & 0.5 & 4.0 & 1.5 \\
\hline Albizzia hasslerii * & 18 & 5.23 & 4.38 & 7.39 & 17.00 & 9.61 & 1.5 & 7.6 & 4.1 & 1.0 & 8.0 & 3.9 \\
\hline Cecropia glaziovii * & 9 & 2.62 & 7.00 & 4.55 & 14.16 & 9.62 & 2.6 & 7.2 & 5.2 & 3.5 & 13.0 & 7.4 \\
\hline Vernonia polianthes & 11 & 3.20 & 5.76 & 2.84 & 11.80 & 8.96 & 2.7 & 7.0 & 5.7 & 3.5 & 9.5 & 6.1 \\
\hline Jaracatia spinosa * & 4 & 1.16 & 5.90 & 1.70 & 8.77 & 7.06 & 7.06 & 3.7 & 6.5 & 4.9 & 14.0 & 10.4 \\
\hline Solanum paniculatum & 7 & 2.03 & 0.42 & 1.70 & 4.16 & 2.45 & 1.2 & 3.8 & 2.7 & 1.0 & 3.5 & 2.0 \\
\hline Cecropia pachystachya * & 4 & 1.16 & 0.72 & 2.27 & 4.15 & 1.88 & 2.0 & 6.2 & 4.2 & 2.5 & 6.0 & 3.5 \\
\hline Ficus sp * & 4 & 1.16 & 0.60 & 2.27 & 4.04 & 1.77 & 1.6 & 3.4 & 2.4 & 2.0 & 4.9 & 3.2 \\
\hline Myrtaceae & 5 & 1.45 & 0.24 & 2.27 & 3.97 & 1.70 & 1.0 & 2.0 & 1.4 & 1.0 & 2.9 & 1.9 \\
\hline Chrysophyllum gonocarpum & 4 & 1.16 & 0.50 & 1.70 & 3.37 & 1.67 & 1.7 & 4.5 & 3.3 & 1.5 & 4.0 & 3.0 \\
\hline Trema micrantha * & 2 & 0.58 & 1.62 & 1.14 & 3.34 & 2.20 & 3.1 & 8.0 & 5.6 & 5.5 & 10.0 & 7.8 \\
\hline
\end{tabular}




\section{REFERENCIAS BIBLIOGRAFICAS}

BERTONI, J. E. de A. Composição florística e estrutura fitossociológica de uma floresta do interior do Estado de São Paulo: Reserva Estadual de Porto Ferreira. 1984. 196 f. Dissertação (Mestrado em Biologia Vegetal) - Instituto de Biologia Vegetal, Universidade Estadual de Campinas, Campinas.

BERTONI, J.E.A. \& DICKFELDT, E.P.,. Plantio de Myracrodruon urundeuva Fr. All. (aroeira) em área alterada de floresta: desenvolvimento das mudas e restauração floresta.2007. Rev.Inst. Flor.,São Paulo, v.19,n.1, p.31-38.

BRASIL. Ministério da Agricultura. Serviço Nacional de Pesquisas Agronômicas. Comissão de Solos. Levantamento de reconhecimento dos solos do Estado São Paulo: contribuição à carta de solos do Brasil. Rio de Janeiro, 1960. (Boletim, 12).

CARPANEZZI, A. A. et al. Espécies pioneiras para recuperação de áreas degradadas: a observação de laboratórios naturais. In: CONGRESSO FLORESTAL BRASILEIRO, 6., 1990, Campos do Jordão. Anais... Campos do Jordão: Sociedade Brasileira de Silvicultura - SBS: Sociedade Brasileira de Engenheiros Florestais - SBEF, 1990. p. 216221.

KAGEY AMA, P. Y.; GANDARA, F. B.; OLIVEIRA, R. E. Biodiversidade e restauração da floresta tropical. In: KAGEYAMA, P. Y. Restauração ecológica de ecossistemas naturais. Botucatu: Fundação de Estudos e Pesquisas Agrícolas Florestais, 2003. cap. 2, p. 27-48.

PICKETT, S. T. A.; COLLINS, S. L.; ARNESTO, J. J. A. Hierarchical consideration of causes and mechanisms of succession. Vegetatio, Dordrecht, n. 69, p. 109-114, 1987. 


\section{Periódica Eletrânica

RODRIGUES, R. R.; GANDOLFI, S. Recomposição de florestas nativas: princípios gerais e subsídios para uma definição metodológica. Rev. Bras. Hort. Orn., Campinas, v. 2, n. 1, p. 4-15, 1996.

VIANA,V. M. Biologia e manejo de fragmentos de florestas naturais. In: CONGRESSO FLORESTAL BRASILEIRO, 6., 1990, Campos do Jordão. Anais... Campos do Jordão: Sociedade Brasileira de Silvicultura - SBS: Sociedade Brasileira de Engenheiros Florestais - SBEF, 1990. p. 113-118.

ZONTA, E.P. \& MACHADO, A.A., 1998. SANEST- Sistema de Análise Estatística.Instituto Agronômico de Campinas, Campinas,S.P. 